XI.

Aus dem pharmakologischen Institut zu Wien.

Untersuchungen zur Physiologie und Pharmakologie des vegetativen Nervensystems.

(II. Mitteilung.)

\title{
Über eine Steigerung der Adrenalinempfindlichkeit
} durch Cocaïn.

Von

A. Fröblich und O. Loewi-

(Mit 2 Kurven.)

Die vorliegende Untersuehung wurde veranlaßt dureh den Wunseh Klarheit über das vielumstrittene Wesen der Cocainmydriasis zu erlangen. Die wohl von den Meisten geteilte Auffassung geht dahin, daß diese Mydriasis Folge einer Reizung der Sympathieusendigungen ist, die den Musculus dilatator iridis versorgen. Die Grundlage dieser Anschauung bildet hauptsäehlich die von Schultz' ${ }^{1}$ ) bestätigte Beobachtung Schölers, wonach wohl noch nach präganglionärer Sympathicotomie aber nicht mehr nach Exstirpation des Ganglion cervicale superius mit folgender Degeneration der postganglionären Sympathieusendigungen die Mydriasis zustande komme. Schon Schultz entschloß sich nur ungern zur Annahme einer Reizwirkung des Cocains als Ursache der Mydriasis, da das Cocain sonst allenthalben - gerade anch am Auge (Einträufeln 5 prozentiger Lösung lähmt die Oculomotoriusendigungen (Schultz) - ein exquisit lähmendes Gift für periphere Nervapparate ist. Nun ist in den letzten Jahren eine Beobachtung gemacht worden, die uns die Möglichkeit zu eröffnen schien, die mydriatische Wirkung des Cocains übereinstimmend mit dessen übrigen, nervlähmenden Wirkungen zu erk]ären. Meltz er ${ }^{2}$ ) hat bekanntlich entdeekt, daß die Exstirpation des Ganglion cerv. sup. die Empfindlichkeit des Dilatators für Adrenalin - für andere Reize hat es Anders on ${ }^{3}$ ) nachgewiesen - eminent steigert.

1) Schuitz: Dubois Arch. 1898. S. 47

2) Meltzer: Amer. journ. of physiol. Bd. 11. S. 28. 1904.

3) Anderson: journ. of physiol. Bd. 30. S. 290. 1904. 
Diese ${ }_{n}$ Sensibilisierung ${ }^{*}$ ist zweifellose Folge eines Wegfalls von Hemmungen der Empfindlichkeit, die durch die Anwesenheit der Nervendigungen ansgetibt werden müssen. Damit ist aber die Annahme möglich gemacht, daß auch die Cocainmydriasis Folge nicht einer Reizung, sondern eines Ausfalls ist, wie die übrigen Cocainwirkungen auch; und zwar könnten wir sie uns entstanden denken durch einen analog wie durch Ganglionextirpation so hier durch Cocain bedingten Ausfall, eine Läbmung von Hemmungen der Empfindlichkeit für normale, periphere sonst unterschwellige Reize, die erst jetzt nach Cocainisierung wirksam werden. Die Tatsache, daß nach Ganglionexstirpation die Mydriasis ausbleibt, würde kein Hindernis für diese Anschaung bilden, da wir wissen, daß biernach der Oculomotoriustonus stark ansteigt und so eine geringfügige Dilatatorkontraktion leicht überkompensieren könnte. Zur Prüfung der Richtigkeit unserer Anschauung von der hemmungsbeseitigenden Wirkung: des Cocains, ergab sich demnach für uns die Aufgabe festzustellen, ob Cocain in der Tat die Empfindlichkeit der Organe für periphere, sympathische Reize, in erster Linie für Adrenalin, steigere. Wir untersuchten in dieser Richtung die Wirkungen des Adrenalins auf Blutgefäße, Harnblase, Auge und Speicheldrüse.

\section{Versuche an Blutgefäben.}

Bekanntlich tubt Cocain selbst eine Wirkung auf Blutgefäße aus und zwar im Sinne einer Konstriktion. Diese Wirkung ist nach eigenen und fremden Erfahrungen wenig konstant und eignet nur größeren Dosen. Bei den von uns in Anwendung gezogenen $(5-10 \mathrm{mg}$ i. v. auf 2-3 kg schwere Kaninchen oder Katzen also 1,6 bis höchstens $5 \mathrm{mg}$ p. Kilo), war sie in zahlreichen Versuchen, über die im einzelnen s. Z. Herr Dr. N eub a u i beriehten wird, nicht ein einzigesmal nachweisbar, auch nicht bei wiederholter Injektion.

Zunächst verglichen wir die Wirkung intravenöser Adrenalininjektionen vor und nach gleichfalls intravenöser Injektion von Cocain in der genannten auf den Blutdruck unwirksamen Dosierung.

Versuch I.

Katze $2 \mathrm{~kg}$ Äthernarkose.

Injektion i. v. Mittl. Blutdruck $\mathrm{mm} \mathrm{Hg}$ Steigerung 0

$0,05 \mathrm{mg}$ Adrenalin

$5 \mathrm{mg}$ Cocain

$0,05 \mathrm{mg}$ Adrenalin
110

170

100

104

190
60

86 
Katze $2,2 \mathrm{~kg}$ Äthernarkose.

Versueh II.

$\begin{array}{cccc}\text { Zeit } & \text { Injektion i. v. } & \text { Blutdruck } & \text { Steigerung } \\ 0 & 0 & 130 & \\ 0 & 0,1 \mathrm{mg} \text { Adrenalin } & \mathbf{2 0 0} & 70 \\ 40^{\prime \prime} & & 120 & \\ 2^{\prime 4} 45^{\prime \prime} & & & \\ 2^{\prime} 45^{\prime \prime} & 0,05 \mathrm{mg} \text { Adrenalin } & \mathbf{1 7 0} & \mathbf{5 0} \\ 3^{\prime} 20^{\prime \prime} & 0 & 100 & \\ 6^{\prime} & 0 & 100 & \\ 6^{\prime} 20^{\prime \prime} & \mathbf{5} \mathrm{mg} \text { Cocain } & & \\ 6^{\prime} 40^{\prime \prime} & & & \\ 7^{\prime} 20^{\prime \prime} & 0,05 \mathrm{mg} \text { Adrenalin } & \mathbf{2 0 0} & \mathbf{1 0 0} \\ 8^{\prime} & & \end{array}$

In beiden Versuchen hat die Injektion von Coeain die Wirkung der daranffolgenden Adrenalininjektion za einer intensiveren gemacht.

Im folgenden Versuch wird der Einfluß einer vorgängigen Cocaininjektion auch auf den zeitlichen Ablauf der Adrenalinwirkung demonstriert :

Katze $1,6 \mathrm{~kg}$ Äthernarkose.

Versueh III.

\begin{tabular}{ccccc} 
Injektion & Blutdruck steigt & \multicolumn{2}{c}{ Steigerungs- } \\
& von & auf & Höhe & Dauer \\
$0,1 \mathrm{mg}$ Adrenalin & $\mathbf{7 2}$ & 124 & $\mathbf{5 2}$ & $\mathbf{1 5 5 ^ { \prime }}$ \\
$0,1 \mathrm{mg}$ Adrenalin & 66 & 122 & $\mathbf{5 6}$ & $\mathbf{1 5 4 ^ { \prime }}$ \\
$\mathbf{5 , 0} \mathbf{m g}$ Cocain & 54 & 50 & 4 & \\
$0,1 \mathrm{mg}$ Adrenalin & 50 & 140 & $\mathbf{9 0}$ & $\mathbf{3 0 0 ^ { \prime }}$
\end{tabular}

Auch in diesem Versuch ist die Blutdrucksteigerung nach vorgängiger Cocainisierung wesentlich höher ausgefallen. Gleichzeitig: hält sie aber auch viel länger an. Letzteres ist ein ganz regelmäßiger Befund. Oft ist sogar der Einfluß des Cocains auf die Dauer der Wirkung viel deutlicher als der auf die Intensität. Versuch IV.

Kaninchen $1,7 \mathrm{~kg}$ Urethannarkose.

\begin{tabular}{ccccc} 
Injektion & \multicolumn{2}{c}{ Blutdruck steigt } & \multicolumn{2}{c}{ Steigerung } \\
& von & anf & Grad & Dauer \\
$0,05 \mathrm{mg}$ Adrenalin & 70 & 124 & 54 & $50^{\prime}$ \\
$0,05 \mathrm{mg}$ Adrenalin & 76 & 130 & $\mathbf{5 4}$ & $50^{\prime}$ \\
$0,05 \mathrm{mg}$ Adrenalin & 84 & 136 & 52 & $49^{\prime}$ \\
$5 \mathbf{m g}$ Cocain & 90 & 90 & & \\
$0,05 \mathrm{mg}$ Adrenalin & 90 & 154 & $\mathbf{6 4}$ & $\mathbf{8 0 ^ { \prime }}$ \\
$0,05 \mathrm{mg}$ Adrenalin & 90 & 154 & $\mathbf{6 4}$ & $\mathbf{9 0}$
\end{tabular}

Dieser Versuch zeigt, daß sich die Wirkung ebenso am Kaninchen wie an der Katze erzielen läßt. Im vorliegenden ist die Wirkung auf die Höhe der Drucksteigerung weit weniger ausgesprochen als die auf die Dauer; docb ist dies kein regelmäßiges Ergebnis. 
Versuch V demonstriert den Einfluß intravenöser Cocaininjektion nach subkutaner Injektion von Adrenalin.

$\begin{array}{ccc}\text { Kaninchen 2,3 } \mathrm{kg} \text { Urethannarkose. } & \\ \text { Zeit } & \text { Injektion } & \text { Blutdruck } \\ 3^{\prime} 45 & 2 \mathrm{mg} \text { Adrenalin s. c. } & 50 \\ 3^{\prime} 50 & & 80 \\ 3^{\prime} 55 & & 95 \\ 4^{\prime}{ }^{\prime} 0 & & 100 \\ 4^{6} 05 & & 90 \\ 4^{\prime} 10 & & 85 \\ 4^{\prime} 11 & 5 \mathbf{5 m g} \text { Cocain i. } \mathbf{~} . & 70 \\ 4^{\prime} 13 & & 155 \\ 4^{\prime} 15 & & 160 \\ 4^{\prime} 16 & & 140 \\ 4^{\prime} 20 & & 90 \\ 4^{\prime} 25 & & 60\end{array}$

Versuch V.

Wie dies bereits Meltzer gezeigt hat, kann auch subkutane Injektion von Adrenalin den Blutdruck unter Umständen beträchtlich steigern. Die Regel ist es nicht und nach unseren Erfahrungen besonders bei Hunden und Katzen ein nur ansnahmsweises Vorkommnis auch bei Verwendung großer Dosen. Wir fanden nun ganz regelmäliig, daß nach dem Abklingen der Drucksteigerung infolge subkutaner Adrenalininjektion, eine darauffolgende intravenöse Injektion einer selbst nicht drueksteigernden Cocaindose so wirkt, wie es Versuch V demonstriert: der Druck beginnt sofort zu steigen, erreicht nach wenigen Minuten sein Maximum, um wieder allmählich zur Norm abzusinken: Es hat ganz zweifellos das Cocain die bereits völlig abgeklungene Wirkung des Adrenalins wieder angefacht. Das "Wie" dieses Vorgangs aber bereitet dem Verständnis nicht geringe Schwierigkeiten. Am nächsten läge anzunehmen, im Blut sei von der subkutanen Injektion her noch ein Adrenalinrest vorhanden, der an sich nicht mehr ausreiche zu wirken aber dadurch wieder wirksam werden könne, daß das Cocain die Adrenalinempfindlichkeit der Gefäßwand steigere. Dieser Deutung widersprechen aber unseres Erachtens 2 Tatsachen: erstlich ist die Blutdrucksteigerung nach Cocain zu beträohtlich als daß sie auf einen ohne Cocain unwirksamen Adrenalinrest obne Künstelei sich beziehen ließe. Ferner aber ist bei dieser Annahme nicht einzusehen, weshalb nicht auch im direkten Anschluf an eine intravenöse Adrenalininjektion Cocain die abgeklungene Blutdrueksteigerung wieder hervorruft: dies ist nach den ersten oben mitgeteilten Experimenten nicht der Fall; und doch läßt sich nach den bekannten und mehrfach bestätigten Versuchen von Harris 
und $W$ eiB 1 ) in diesem Moment Adrenalin im Blut deutlich nachweisen. Gerade diese Differenz im Verhalten der Cocainwirkung je nachdem eine inträvenöse oder eine subkutane Adrenalininjektion vorausgegangen ist, läßt uns für den Moment an einer zureichenden Deutung verzweifeln uud wir müssen uns mit der zunächst interessierenden Feststellung begnügen, daß das Cocain in an sich nicht blutdrucksteigernder Dosierung die Adrenalinwirkung mäehtig steigert.

\section{Versuche am Auge.}

Bei diesen Versuchen mußte mit besonderer Vorsicht zu Werke gegangen werden, da das Cocain bekanntlich selbst mydriatiseh wirkt. Wir mußten daher eine ev. adrenalinsensibilisierende Wirkung auf verschiedenen Wegen festzustellen suchen.

\section{Versuch VI.}

5 Kaninchen erhielten je einen Tropfen 1 prozentige Cocainlösung in ein Auge eingeträufelt: Es trat weder Anästhesie noch Mydriasis ein. Am nächsten Tage wurde den gleichen Tieren um 10 Uhr $35^{\prime}$ je 2 Tropfen Adrenalin $1 \%$ links instilliert. Nach 20 Nhin. war bei zweien eine deutliche Mydriasis eingetreten. Sie schieden aus dem Versuch aus. Bei den drei übrigen war auch um 11 Uhr 25', mithin 50 Min. nacu der Adrenalininstillation eine Mydriasis nicht eingetreten. Sie erhielteh darum jetzt je einen Tropfen der bei ihnen ja unwirksamen 1 prozentigen Cocainlösung in beide Augen. Um $12 \mathrm{Uhr} 10^{\prime}$ war bei sämtlichen die linke Pupille maximal mydriatisch. Eshattealsodas Cocain eine an sich unwirksame Adrenalindose wirksam gemacht.

Versuch VII.

Eine Katze erhält i. v. $0,25 \mathrm{~g}$ Urethan.

Wiederholte intravenöse Injektion von $0,02 \mathrm{mg}$ Adrenalin ist ohne Einfluß auf die Pupillenweite. Jetzt werden $5 \mathrm{mg}$ Cocain intravenös injiziert: Pupillen werden mittelweit. Neuerliche Injektion obiger Adrenalinmenge ruft jetzt maximale Mydriasis hervor.

\section{Versueh VIII.}

Bei Hunden wirkt Adrenalin intravenös injiziert in der Regel nicht mydriatisch, wie wir in Bestätigung der Angaben von Elliott2) fanden.

Einem Hund wurde in ein Auge 4 Tropfen Cocain 2 proz. instilliert: Mydriasis, die sich beim Hund wie auch bei der Katze keineswegs

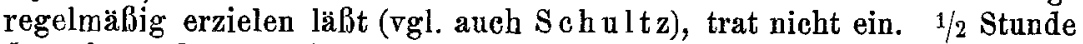
danach wurde $1 \mathrm{mg}$ Adrenalin i, v. injiziert: das cocainisierte Auge und zwar dies allein, wurde mydriatisch. Der Versuch bei Katzen durch Instillation an sich nicht mydriatisch wirkender Cocain. lösung das Auge für nachfolgende subkutane Adrenalininjektion wie nach Ganglionexstirpation empfindlich zu machen, schlug jedesmal fehl. Vielleicht reicht hierzu die zur Resorption gebrachte Cocainmenge nicht aus.

1) Harris u. Weiß. Pflügers Archiv. Bd. 103. S. 510. 1904. vgl. auch Ehrmann: Arch. f. exp. Pathol. u. Pharm. Bd. 56. S. 98. 1905.

2) Elli ott: journ. of physiol. Bd. 32. S. 290. 1904. 
Die Gesamtheit dieser Versuehe seheint wohl daftr zu sprechen, dab das Cocain für die Adrenalinwirkung auch am Auge sensibilisiert.

III. Versuche an der Harnblase der Katze.

Wir prüften in Vorversuchen, ob nach Anwendung der von uns benutzten Cocaindose ein stärkerer Strom zur Erzielung des Pelvicusschwelleneffektes nötig ist als vorher. Wir überzeugten uns davon, daß dies nicht der Fall ist. Hiernach haben wir bei der Katze in bekannter Weise die Blase mit einem Wassermanometer und dies mit einem Pistonrecorder verbunden, dessen Schwankungen am Kymographion verzeichnet wurden. Bei dem Versuch gingen wir dann so vor wie bei den Blutdruekversuchen d. h. wir registrierten den Erfolg: der Adrenalininjektion vor und nach Injektion von $5 \mathrm{mg}$ Cocain. Wir haben nun in wiederholten Versuchen festgestellt, daß ebenso wie die Blutdruckwirkung des Adrenalin, so auch seine blasenerschlaffende durch vorgängige Cocainisierung wesentlich gesteigert wird, sowohl nach Intensität wie nach Dauer und zwar letztere in gleichem Ausmab wie der Blutdruck. Zur Illustration des Gesagten dienen die folgenden Kurven: die erste (Kurve I) zeigt die Blasenerschlaffung nach Adrenalin allein, die zweite (Kurve II) nach vorgängiger Cocaininjektion ${ }^{1}$ ).

Also nicht nur die fördernden, sondern auch die hemmenden Wirkungen von Adrenalin werden dureb Cocain sensibilisiert.

IV. Versuche an der Speicheldrüse.

Es erïbrigte, nachdem wir bisher nur muskuläre Wirkungen des Adrenalins nntersucht hatten, nunmehr zu prïfen, ob etwa auch seine sekretorischen Wirkungen, wie es eigentlich a priori anzunehmen war, durch Cocain eine Steigerung erfahren würden. Als Objekt wählten wir die Unterkieferspeicheldrüse der Katze. Die Präparation und Registration geschah in der von uns nun mehrfach geschilderten Weise.

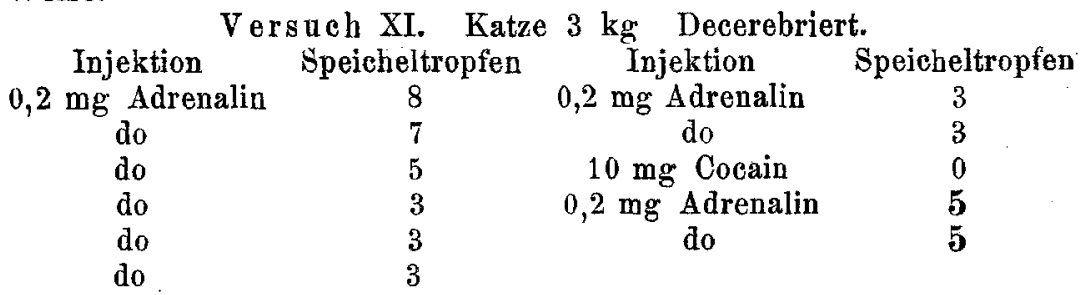

1) Elliott. loc. cit. S. 411, fand, daß die Blase ebenso nach Denervierung hauptsächlich länger aber nicht stärker auf Adrenalin reagierte. 
Kurve II.

Kurve I.
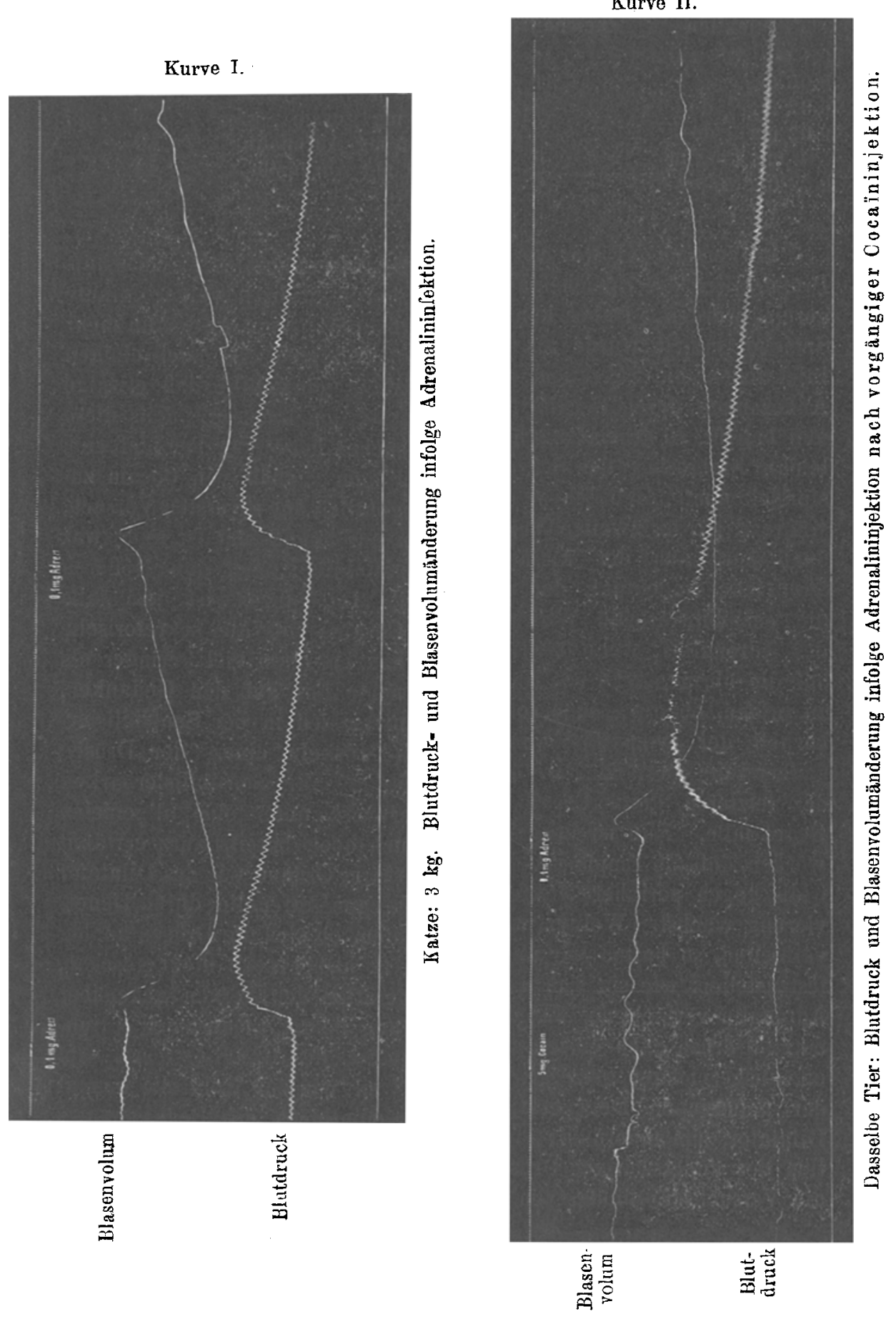
Versuch XII. Katze $3,5 \mathrm{~kg}$. Decerebriert.

\begin{tabular}{cclc} 
Injektion & Speicheltropfen & \multicolumn{1}{c}{ Injektion } & Speicheltropfen \\
$0,1 \mathrm{mg}$ Adrenalin & 16 & $0,02 \mathrm{mg}$ Adrenalin & 4 \\
$0,05 \mathrm{mg}$ do & 10 & $0,05 \mathrm{mg}$ Adrenalin & $\mathbf{1 2}$ \\
$0,02 \mathrm{mg}$ do & 2 & $10 \mathrm{mg}$ Cocain & 0 \\
$0,02 \mathrm{mg}$ do & 3 & $0,05 \mathrm{mg}$ Adrenalin & $\mathbf{1 0}$ \\
$10 \mathrm{mg}$ Cocain & 0 & $0,1 \mathrm{mg}$ Adrenalin & $\mathbf{1 4}$ \\
$0,02 \mathrm{mg}$ Adrenalin & $\mathbf{4}$ & &
\end{tabular}

Im Versuch XI war vor Beginn der Adrenalininjektionen behufs Präparation des Speichelgangs die Chorda während einer Minute gereizt worden. Daher kommt es, daß anfangs mit Adrenalin mehr Speichel erzielt wurde als später und daß sich erst nach einigen Injektionen. die Sekretion so gleichmälig einstellte, dab etwaige Änderungen durch Cocain festgestellt werden konnten. Was diese nun betrifft, so scheint aus beiden Versuchen hervorzugehen, daß eine leichte Steigerung der Sekretion unter dem Einfluß von Cocain zustandekommt. Deutlich ist sie aber eigentlich nur im ersten Versuch, während sie im zweiten Versuch eben nur angedeutet ist und zwar auch nur nach der kleinsten Adrenalingabe; nach den größeren fehlt jede Andeutung der Steigerung. Im ganzen ist jedenfalls die sensibilisierende Wirkung des Cocains auf die sekretorische des Adrenalins, wenn tuberhaupt vorhanden, dann viel geringer als auf die ubrigen. Dieses Ergebnis brachte uns auf den Gedanken, zu prüfen, ob etwa auch sonst ein Unterschied in der Sensibilisierbarkeit der sympathisch innervierten Muskeln einer- der Drüsen andererseits bestände, so dab die mangelhafte Sensibilisierbarkeit der letzteren durch Cocain möglicherweise unter eine allgemeine Regel falle. Non ist bekannt und auch oben bereits kurz erwähnt worden, daß die Empfindlichkeit muskulärer Organe wie des Dilatator iridis gegen periphere Reize so auch gegen Adrenalin durch Denervierung wächst. Wollten wir also prüfen, ob sich die Drïsen analog verhielten, so hatten wir nur, was $u$. E. bisher noch nicht geschab, festzustellen, wie sie sich nach Degeneration der Nerven gegenüber Adrenalin verhielten. Für diese Prüfung liefert wiederum die Submaxillardrüse nach einseitiger Exstirpation des Ganglion cerv. sup. ein ausgezeichnet günstiges Objekt, weil wir zur Kontrolle einmal der gelungenen Degeneration des Sympathicus die Reaktion des Dilatator, zum andern der etwa gesteigerten Empfindlichkeit die Sekretion der andersseitigen Drüse verfolgen können. Hieraus ergab sich die Versuchsanordnung von selbst. 
Katze $2 \mathrm{~kg}$.

\author{
Versueb XIII.
}

21. 5. Rechtes Ganglion cerv. sup. exstirpiert.

28. 5. Adrenalin $1 \mathrm{mg}$ subkutan injiziert. Nach einer Stunde rechts maximale Mydriasis, linke Pupille unverändert.

30. 5. Äthernarkose. Speichelgänge beiderseits mit Kanülen armiert, Ablesung der ausfließenden Speichelmengen, die in graduierten Kanülen vorrücken.

Zeit Injektion

12 Uhr 12 Min.

$\begin{array}{llll}12 & " & 12 & " \\ 12 & " & 14 & " \\ 12 & " & 17 & " \\ 12 & " & 21 & " \\ 12 & \prime & 22 & " \\ 12 & " & 25 & " \\ 12 & " & 27 & " \\ 12 & " & 28 & " \\ 12 & " & 29 & " \\ 12 & " & 30 & " \\ 12 & " & 35 & "\end{array}$

0

$0,5 \mathrm{mg}$ Adrenalin

0,5 mg Adrenalin

$2,5 \mathrm{mg}$ Atropin

$0,5 \mathrm{mg}$ Adrenalin

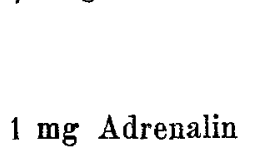

Speichel rückt vor um mm rechts links

0

0

$\begin{array}{rr}15 & 14 \\ 2 & 7 \\ 21 & 31\end{array}$

Speichelfluß steht

$\begin{array}{ll}8 & 8 \\ 3 & 5 \\ 5 & 7\end{array}$

$14 \quad 16$

Die Tatsache, daß am 7. Tag nach der rechtsseitigen Ganglionexstirpation Adrenalin auf der gleichen Seite mydriatisch wirkte, beweist, dah die Degeneration der Sympathicusendigungen vollständig und der Dilatator überempfindlich gegen Adrenalin geworden war. Ganz im Gegensatz hierzu kann nach dem Ergeonis unseres Versuches von einer gesteigerten Empfindlichkeit der Speicheldrüse nicht die Rede sein: der Erfolg der Adrenalininjektion war auf beiden Seiten völlig identisch. Besonders schön zeigt sich dies nach Atropinisierung, durch die wir eine etwaige die Klarheit des Ergebnisses trübende Beteiligung der Chorda ausgeschlossen. Damit ist aber der Nachweis geliefert, daß die Überempfindliehkeit gegen periphere Reize als Folge degenerativer Denervierung bei Drüsen sich nicht einstellt und somit anch die wahrscheinliche Erklärung für die relative Wirkungslosigkeit des Cocains quoad Adrenalinsensibilisierung an diesen Organen gegeben. Das Ergebnis unseres Versuches steht in guter Harmonie zu der Tatsache, daß nur nach Durchtrennung der Chorda nicht aber nach der des Sympathieus eine sog. "paralytisehe Sekretion auftritt, die wohl allgemein als Folge gesteigerter Empfindlichkeit der Drüse analog der paradoxen Pupillenreaktion anfgefalit wird. Darnach bestände also eine wesentliche Differenz in dieser Beziehung $z$ wischen autonomem und sympatbischem Nerv. Fassen wir das Bisherige zusammen, so können wir also sagen, 
daß das Coeain überall da, wo therhaupt eine Sensibilisierung möglich ist, d. i. an den muskulären Organen, die Wirkung des Adrenalins als eines spezifisch sympathischen Giftes sensibilisiert. Wir hatten nur noch festzustellen, ob diese Sensibilisierung sich wirklich nur auf sympathische Gifte bezieht oder auch autonome betrifft. Wir haben einen derartigen Versuch ausgeführt und zwa: prüften wir die Beeinflussung der Wirkung des antonom reizenden Pilocarpins durch Cocain.

\section{Versuch XIV.}

Katze $3 \mathrm{~kg} 1 / 4 \mathrm{~g}$ Urethan i. v. Beide Vagi durchschnitten, künstliche Atmung.

\begin{tabular}{ccc} 
Injektion & \multicolumn{2}{c}{ Blutdruckfall } \\
& von & auf \\
$0,2 \mathrm{mg}$ Pilocarpin & 120 & 80 \\
do & 130 & 100 \\
$10 \mathrm{mg}$ Cocain & & \\
$0,2 \mathrm{mg}$ Pilocarpin & 130 & 100 \\
do & 130 & 100
\end{tabular}

Der Versuch zeigt deutlich, daß das Cocain bei der von uns gewählten Dosierung auf die Wirkung eines autonomen Giftes ohne jeglichen Einfluß ist.

\section{Diskussion der Ergebnisse.}

Wir haben im Bisherigen die von uns beobachtete Cocainwirkung als "Sensibilisierung" fur Adrenalin bezeiehnet. Damit wollen wir zum Ausdruck gebracht haben, daß es sich bei der kombinierten Cocain-Adrenalinwirkung nicht um eine Summation handele; eine solehe schließen wir darum aus, weil die von uns angewandte Cocaindose nach unsern eigenen und nach fremden (v. Anrep ${ }^{1}$ ), U. Mosso2) Versuchen weit unterhalb der wirksamen Dose liegt. Für die Annahme einer Summation wäre ferner Vorbedingung, daß die peripheren Wirkungen des Cocains denen des Adrenalins gleichsinnig wären. Nun sind wir aber über diese Wirkungen ganz ungenügend unterrichtet; nicht nur, daß die Angaben der verschiedenen Autoren hiertiber voneinander abweichen, auch die Analyse darauf hin, ob es sich um peripheren oder zentralen Ursprung handele, ist außer bei der Mydriasis nicht systematiseh durchgeführt. Aus diesem Grunde sind wir auch außerstande zu sageu, ob die Erscheinungen der Vergiftung mit größeren Cocaindosen im Gebiete der vegetativen Organe u. zw. insbesondere der sympathisch innervierten, also die Gefäßkonstriktion und die Mydriasis, Folge oder alleinige Folge

1) v. Anrep: Pflügers Arch. Bd. 21. S. 38. 1850.

2) U. Mosso: Arch. f. exp. Path. u. Pharm. Br. 23, S. 153. 1887. 
davon sind, daß unter dem Einfluß von Cocain sonst unwirksame, physiologiseherweise vorhandene Adrenalinmengen zur Wirkung: kommen. Was die Mydriasis im besonderen anlangt, so könnte, wie wir bereits erwähnt haben, die Tatsache a priori gegen den eben angeführten Mechanismus sprechen, daß sie nach Exstirpation des Ganglion cerv. ausbleibt, während die Wirkung von Adrenalin erhalten bleibt, demnach auch die adrenalinsensibilisierende des Cocains in die Erscheinung treten mübte. Wir baben auch bereits erwähnt, daß das Erstarken des Oenlomotoriustonus infolge Exstirpation des Ganglions Ursache dieses Ausfalls sein könnte. Daß in der Tat die Möglichkeit der Cocaineinwirkung am Auge mit der Ganglionexstirpation nicht entfällt, geht aus versehiedenen Beobachtungen hervor. Einmal fand Limbourg ${ }^{1}$, daß auch nach diesem Eingriff die Cocainmydriasis noch lange zustande kommt, wofern vom Moment der Operation an das Auge dauernd unter Cocainwirkung gesetzt wird. Wir deuten dies so, daß hierdureh der Oculomotoriusibertonus an seiner Entwicklung gehemmt wird. Ferner fand er, daß am ganglienlosen Auge im Anschluß an eine Cornealelektrisierung eine Cocainmydriasis hervorgerufen werden kann; diese Beobachtung kann kaum anders gedeutet werden, als daß das Cocain für einen durch die Elektrisierung gesetzten Nachreiz empfindlich gemacht hat 2). Trotzdem scheint es uns noch nieht erlaubt zu sein in der von uns entdeckten Wirkung des Cocains die einzige Ursache der Cocainmydriasis zu sehen.

\section{Zusammenfassung.}

An sich ganz unwirksame Gaben von Cocain steigern in hohem Maß die Adrenalinwirkung und zwar nach Intensität wie Dauer. Festgestellt wurde dies für die Wirkung auf BlutgefäBe, Harnblase und Ange. Die Tatsache, daß die blutdrucksteigernde Wirkung des Adrenalins nach subkutaner Injektion erst bei gleichzeitiger Anwendungkleinster, an siehselbstganzwirkungsloser Cocaindosen sicher hervorgerufen, mindestens nach Intensität und Dauer enorm gesteigert werden kann, fordertzurAnwendung dieser Kombination in der Praxis auf.

1) Limbourg: Arch. f. exp. Path. u. Pharm. Bd. 30. S. 93. 1892.

2) Kochmann fand nach geringen Cocaindosen eine gesteigerte elektrische Erregbarkeit des Myocard. Arch. int. de pharmacodyn. Bd. 18. S. 41. 1907.

Das Analogon zu diesem Versuch ist der von Schultz: Eserinmiose im Anschluß an Cornealreizung nach degenerativer Entfernung des Ciliarganglions. 